\title{
Phase Based Localization for Underwater Vehicles Using Interval Analysis
}

\author{
Mohamed Saad IBN SEDDIK, Luc JAULIN and Jonathan GRIMSDALE
}

\begin{abstract}
This paper addresses the common issue of locating an Underwater Vehicle (UV). Usually, the positioning of a vehicle is based on the propagation of electromagnetic waves, using systems such as the Global Positioning System (GPS). However, water, and particularly salty water, makes the use of electromagnetic waves impractical due to the attenuation caused by the high conductivity of the medium. Hence, the most reliable way to transmit information underwater is by using sound waves and many technologies have emerged to solve the positioning problem in this way. Technologies such as Long BaseLine (LBL), Short BaseLine (SBL) and Ultra-Short BaseLine (USBL) are the most frequently used underwater. These technologies are based on the use of multiple emitters in the case of LBL and SBL, or multiple receivers in the case of USBL. This paper describes a way of finding a vehicle location, on-board, based on the measurement at the vehicle by a single receiver of the phase of an acoustic sine wave transmitted from a single emitter that is at a fixed and known location. The method also uses other proprioceptive measurements: vehicle's velocity and heading. An algorithm based on contractors and bisections scatters the solution space searching for all possible solutions (positions in this case) to the set of equations. Moreover, this paper introduces the Time Constraint Satisfaction Problem (TCSP). Indeed, the proposed algorithm does not compute the solutions from measurements at a single point in time, rather it uses a set of measurements taken over a time window and stored in a buffer. As a result, the location is not only known at the latest instant but the past locations can be tracked back over the length of the chosen time window.
\end{abstract}

Keywords. Interval Analysis, Acoustic Localization, Underwater Localization, GPS, USBL, LBL, Time Constraint Satisfaction Problem, TCSP, Constraint Satisfaction, CSP, Contractor, Sequential Monte Carlo, Particle Filters.

\section{Introduction}

In this paper, we are interested in finding a solution to a localization problem that is difficult to solve using conventional methods.

Underwater, the localization of objects is not easy due to the high conductivity of water, and particularly salty water, that attenuates electromagnetic waves and makes them impractical to use. So for this kind of environment, it is very common to use sound waves to communicate and localize objects.

Previous works have found different ways to use sound for the localization issue either by using multiple sources and unique receiver like the Long Baseline [19], or a unique source and multiple receivers on the vehicle like the Short Baseline or the Ultra-Short Baseline [17, 18]. All these solutions rely on having multiple acoustic paths to compute the position. For example, LBL uses multiple sources and one receiver and SBL as well as USBL use one source and multiple 
receivers. However, in a marine environment, the less equipment deployed the lower is the risk of malfunction.

We shall be introducing in this paper a new technique that propagates recorded information about a problem through time in order to solve it. The technique is called Time Constraint Satisfaction Problem and it is an evolution of Constraint Satisfaction Problem [10] that now uses time too to solve the system. It differs from the techniques developed in [5] as a history (or buffer) only contains proprioceptive data and keeps no track of previously calculated solutions. The application of TCSP presented here relies on only one source and one receiver and uses other sensors already on-board most UVs such as a compass and optionally a Doppler Velocity Log (DVL). We shall be using this example to prove that the algorithm is able to position the UV in extreme situations with very little information. Unlike a SLAM problem [8], this method is only used for localization and not for mapping. The source of the signal is considered as the origin of the reference frame and the position is only resolved using the recorded information, in this case, a single sound wave.

The problem studied is as follows, an UV is at an unknown position underwater. Using an embedded hydrophone, it records acoustic signals within the bandwidth of a continuous sinusoidal waveform that is emitted from a source that is considered to be the origin of the reference system. we shall develop in section $\S 2$ the theory and the mathematical background from the equations to the TCSP algorithm. The motivation is explained in the section $\S 3$. Finally, the application and results are in section $\S 5$ before the conclusion and future work in section $\S 6$. In addition to Kieffer's approach [12], this method provides not only outer contraction but also inner contractions [4].

\section{Mathematical Background}

\section{Acoustic signal}

As a starting point of the problem, we suppose that the source of the sine wave (SW) emits a pure tone at a given frequency $f$. The generated signal can be represented as follows:

$$
s_{\text {source }}=A_{\text {source }} \cdot \sin (\omega \cdot t),
$$

with $A_{\text {source }}$ describing the amplitude of the sine wave and $\omega=2 \pi \cdot f$ representing the angular velocity of the SW.

At an unknown distance $d$, an UV is in motion. It is using its on-board sensors to measure $s$ the distorted signal that is received. At the UV position, the recorded signal can be represented by:

$$
s=A \cdot \sin (\omega \cdot t-\varphi),
$$

where $A$ describes the amplitude ${ }^{1}$ of the signal, $\omega$ represents the angular velocity and $\varphi$ describes the phase-shift between the original signal and the recorded signal.

Information about the distance is actually stored in the phase-shift as:

$$
\varphi=\omega \cdot \frac{d}{c}
$$

where $c$ is the celerity of the wave in the environment and $d$ is the distance from the source. $d$ can be written $\operatorname{as}^{2} d=\sqrt{p_{x}^{2}+p_{y}^{2}}$ with $\left(p_{x}, p_{y}\right)$ are the coordinates of the UV.

Equation (2.3) can be then rewritten as :

$$
\varphi=\omega \cdot \frac{\sqrt{p_{x}^{2}+p_{y}^{2}}}{c},
$$

\footnotetext{
${ }^{1}$ For the sake of simplicity, the amplitude is considered equal to one. In real situation, the attenuation of the signal requires amplifiers and filters to recover the waveform.

${ }^{2}$ Considering that the UV and the source are in the same plane, depth is not taken into account.
} 


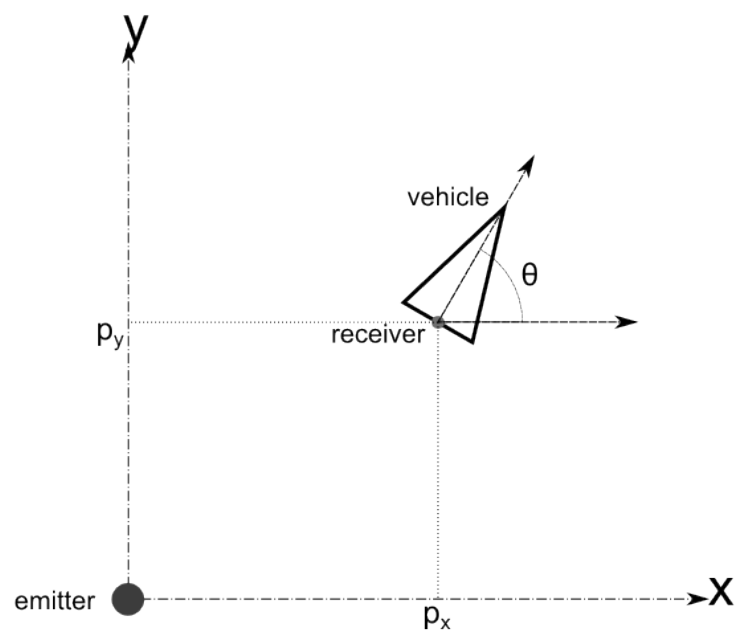

FIGURE 2.1. AUV is at $\left(p_{x}, p_{y}\right)$ from the emitting source.

and then Equation (2.2) becomes:

$$
s=\sin \left(\omega \cdot\left(t-\frac{\sqrt{p_{x}^{2}+p_{y}^{2}}}{c}\right)\right) .
$$

Note that the useful information is contained in the phase, Equation (2.4). However, instead of using usual phase detection techniques $[13,6]$, we propose to use the signal as recorded and process the amplitude ${ }^{3}$.

\section{Equations}

For the UV state equations, we suppose that it has a unicycle model where $\left(p_{x}, p_{y}\right)$ are the coordinates of the UV, $\theta$ its heading and $v$ its velocity ${ }^{4}$. We then have all the equations that constitute our problem to solve represented in 2.6.

$$
\left\{\begin{array}{l}
\dot{p_{x}}(t)=v(t) \cdot \cos \theta(t) \\
\dot{p_{y}}(t)=v(t) \cdot \sin \theta(t) \\
s(t)=\sin \left(\omega \cdot\left(t-\frac{\sqrt{p_{x}^{2}+p_{y}^{2}}}{c}\right)\right)
\end{array}\right.
$$

Let's put $x=\left[p_{x}, p_{y}\right]^{T}$ and $f: \mathfrak{R}^{2} \rightarrow \mathfrak{R}^{2}$. The Equation (2.6) can be rewritten

$$
\left\{\begin{array}{l}
\dot{x}(t)=f(u(t)) \\
s(t)=\sin \left(\omega \cdot\left(t-\frac{\sqrt{p_{x}^{2}+p_{y}^{2}}}{c}\right)\right),
\end{array}\right.
$$

with $u(t)=[v(t), \theta(t)]^{T}$.

\footnotetext{
${ }^{3}$ A filter and an amplifier might be needed to recover most of the signal. Note that, thanks to intervals, it is not needed to have a perfect SW recorded.

${ }^{4}$ The velocity can be measured or deduced using a mapping between the desired thrust and interval taking into account currents.
} 
As $f$ does not depend on $x$ we can integrate the Equation (2.7) directly [2]:

$$
\left\{\begin{array}{l}
x(t)=\int_{t_{0}}^{t} f(u(\tau)) d \tau \\
s(t)=\sin \left(\omega \cdot\left(t-\frac{\sqrt{p_{x}^{2}+p_{y}^{2}}}{c}\right)\right) .
\end{array}\right.
$$

\section{Motivations}

The problem described previously is non-linear and no obvious solutions can be suggested. Because of the sine, it is understood that the set of solutions is an infinite number of concentric circles that have the same phase. To avoid investing in high cost additional equipment, the suggested method should take advantage of already available navigation data as the velocity provided by the DVL or other speed measurement tool, and the heading of the vehicle provided by the embedded compass. The method should also guarantee that no possible position is missed to maximize reliability. It should also be robust against three major issues that can be faced by an underwater vehicle:

\section{Kidnapping}

During a mission, an UV faces many unexpected phenomena: a sudden strong undetected gust of current, a loss of signal or total system blackout. All these effects can be grouped under kidnapping [7] as the UV has no clue about what happened to it. The method presented here counters any kidnapping effect by resetting the position boxes to $[-\infty, \infty]$ at the beginning of each computation giving the method the quality of forgetfulness. Some may consider using the previous computed boxes, however, in the case of undetected outliers, the set of solutions will be altered and relaxed intersection $[9,15]$ maybe needed. The presented method, on the other hand, will still be able to retrieve more precise position once the outliers are discarded. Also, whenever the set of solution boxes is empty, it is certain that outliers has been introduced in the buffer and the buffer needs to be flushed to have a new set of solution boxes.

\section{Unknown Starting Point}

Another advantage of the method should be the ability to start the localization process with no constraints about the starting point [16]. The algorithm should be able to localize the UV no matter when it is turned on and does not need a infinite history as we shall show in section $§ 5$. It also means that the UV does not need to surface and interrupt its mission to relocate itself as required when using an Inertial Navigation System that needs a GPS fix periodically to avoid errors that are integrated into the position estimation or by any other similar methods.

\section{Path Reconstitution}

Finally, the method provides the ability to rebuild the path of the UV for the length of the buffer and not only the final position. This feature is useful when the mission of the UV is to map the sea floor or locate a lost target on the sea bottom. The time propagation technique explained later updates the position box through time (path reconstruction) until the desired accuracy is reached.

\section{Algorithm}

The algorithm proposed in this paper aims to solve a set of equations with multiple variables changing over time, which can be considered as a generic Numerical CSP[1] where time has effect on each variable.

To find a solution to Equation (2.8), two forward-backward ${ }^{5}$ contractors $C^{\int}$ and $C^{s}[4,3]$ are proposed. We understand that the only variable to be contracted is $x$. All the other terms are

${ }^{5}$ Forward-backward contractors are known the be very efficient for similar constraints problems. 


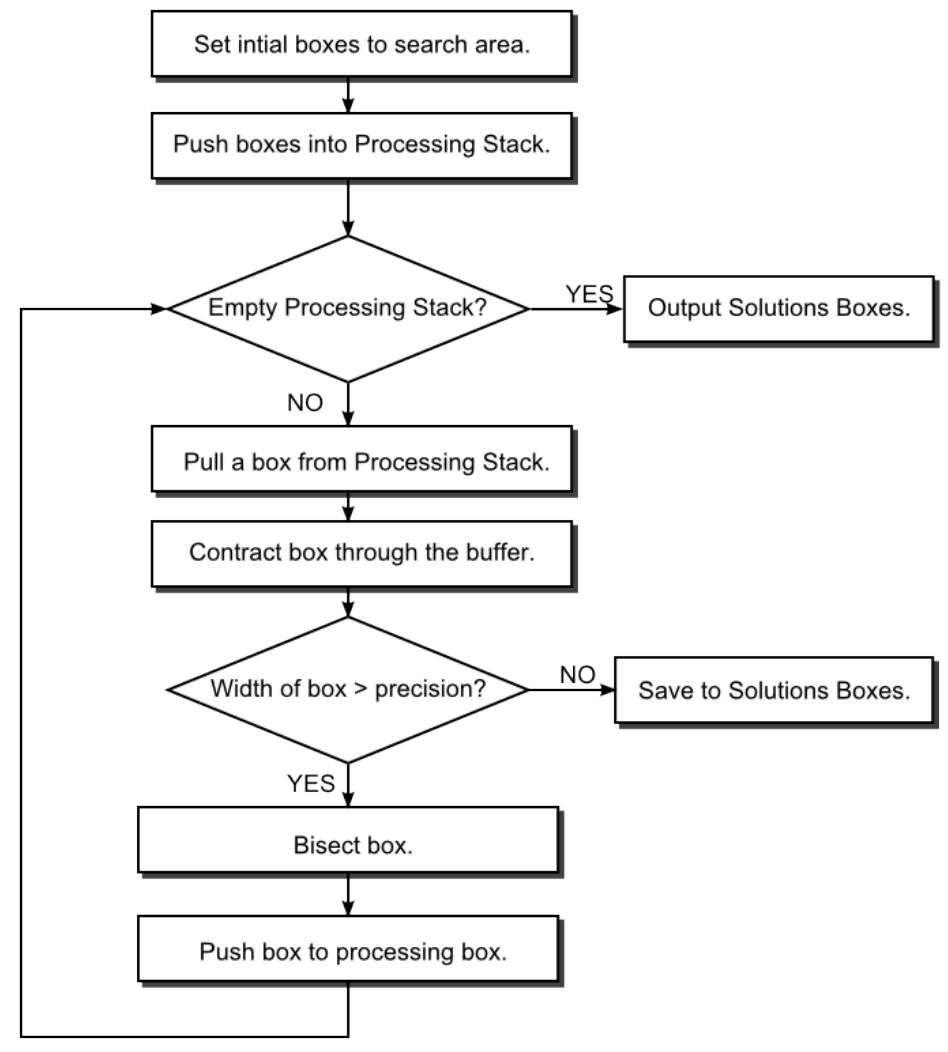

FIGURE 4.1. Algorithm

either measured or deduced. A contractor is a operator from $\mathfrak{R}^{n} \rightarrow \mathfrak{R}^{n}$ that excludes intervals not respecting the constraints defining the contractor.

Consider a First-In First-Out buffer (FIFO) of limited length $N$ that will be fed with $u$ (speed and heading) and $s$ (amplitude of the received signal). As no information is provided about the starting point, the initial intervals for $x=\left[p_{x}, p_{y}\right]^{T}$ are $[[-\infty, \infty],[-\infty, \infty]]^{T}$.

The main idea behind the TCSP algorithm, figure 4.1, is to propagate the information only within the FIFO while having an approach similar to the Set Inversion Via Interval Analysis (SIVIA) described in $[8,11]$.

The proposed algorithm suggests to reset all the boxes to the global search area. This is mandatory to robustify the localization algorithm against kidnapping as explained in subsection 3. This reinitialization allows the algorithm to perform better by not integrating errors over time, explaining the choice of finite-length FIFO. The initialized boxes are then put into the stack of the boxes to be processed. Each box $x$ of the stack is pulled from the stack and contracted using $C^{\int}$ and $C^{s}$ through the buffer propagating information. Once all the buffer has been used to contract the $x$ box, its width evaluated for the required precision. If the width meets the precision, the contracted box is saved to the solutions boxes set, else the box is bisected into two boxes and pushed to the stack for further investigations. 


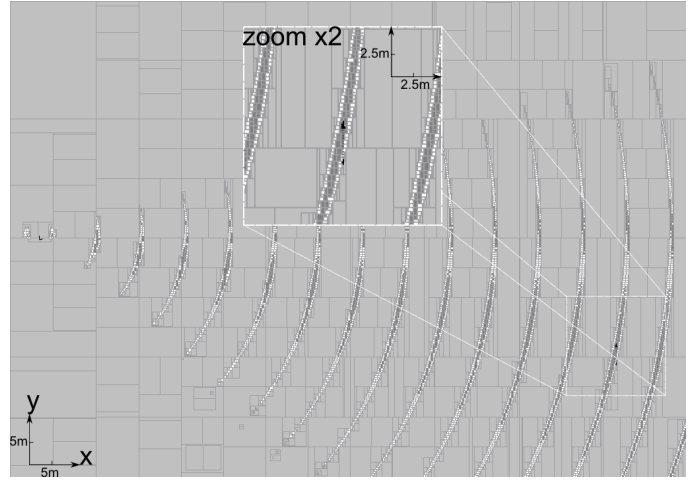

(A) Buffer 300 milliseconds

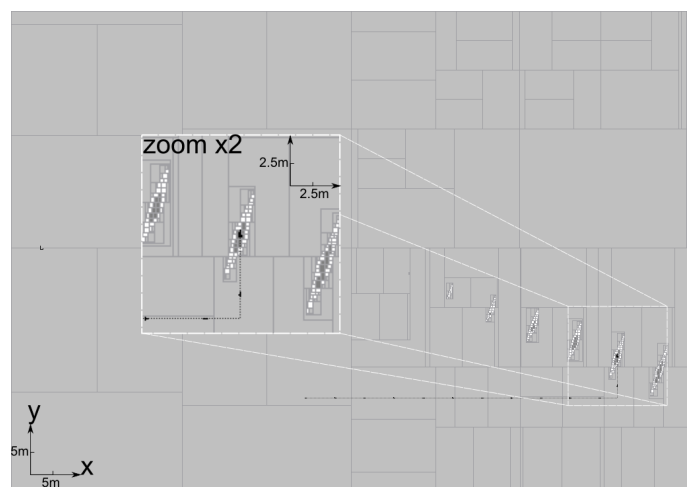

(C) Buffer 6 seconds

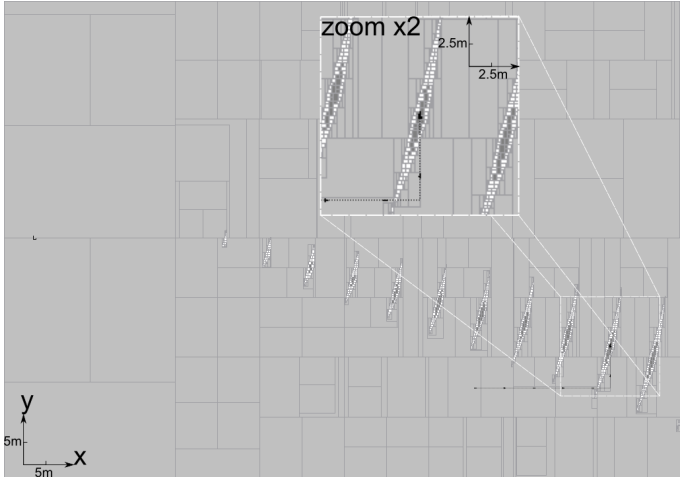

(B) Buffer 3 seconds

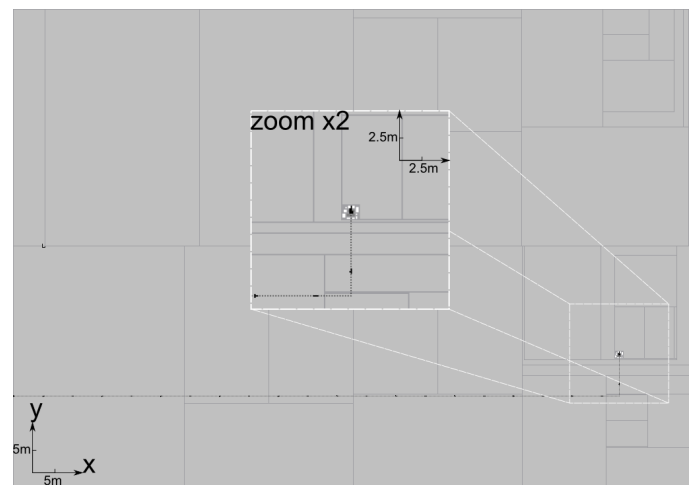

(D) Buffer 12 seconds

FIGURE 5.1. Results

\section{Applications}

We propose the following simulated scenario to test our algorithm: an UV starts from an unknown position recording data from the DVL (speed), the compass (heading) and the hydrophone (acoustic signal). The generated set of data is given to a software running the previously described algorithm to localize the UV.

The drawing convention for figure 5.1 is that intervals that are solution to the TCSP are drawn in dark gray, those that are not a solution are drawn in light gray and the white colored intervals that are perhaps a solution. Inner and outer contractors [4] are used to differentiate between intervals that are perhaps a solution and the intervals that represent a solution. In real-life, we might only be interested in perhaps intervals which can reduce the computing time as we will only apply the outer contractor. The UV is represented with a little black triangle and the source is represented by the little $\llcorner$ in the center of the crescents.

For the first figure 5.1a, the buffer can be considered as empty as it only contains 300 samples $^{6}$ (one sample each millisecond) that are insignificant for the wave length chosen $(1 \mathrm{kHz})$. Notice that the algorithm is not capable of solving the position with such few data. In figure $5.1 \mathrm{~b}$ and figure $5.1 \mathrm{c}$, at the same position but with longer buffer and more data are available, fewer solutions are found.

\footnotetext{
${ }^{6}$ The doted trail behind the AUV (represented by a triangle) shows the size of the buffer.
} 
Finally in figure 5.1d, the algorithm contracts the space to small boxes around the UV when the buffer contains enough data.

\section{Conclusions And Future Work}

We have shown that the presented method is capable of solving a deterministic non-linear system. The main advantages explained in section $\S 3$ are critical for some systems, particularly underwater vehicles. However, the use of the full space as a starting interval can be computationally heavy. For the simulated test case explained in section $\$ 5$ as an example, a single core spends more than 30 seconds to find the solution for a 6 seconds-long buffer. However, as interval analysis can be easily parallelized, the same example finishes in less than 5 seconds with 8 cores. Future work will consist in testing the algorithm with field recorded data and will use more realistic inflation for the interval. It is important to note that even a very low inflation for the state variables makes the inner contractor useless which is not a major issue for localization as we only need to know where we probably are, which can be provided by the outer contractor.

On the other hand, this method only provides a relative positioning system. Some may suggest embedding information about the absolute position of the source within the signal. Also, with the environmental noise, the use of a Fourier transform will be more reliable than the amplitude of the signal to determine the phase shift. But again, the amplitude was used in section §2 just to prove that this method is capable of resolving a complex non-linear system.

A comparison with similar techniques, such as particle filters or sequential Monte Carlo [14], could reveal the usefulness of this method compared to probabilistic methods.

\section{References}

[1] Ignacio Araya, Bertrand Neveu, and Gilles Trombettoni. Exploiting common subexpressions in numerical csps. In PeterJ. Stuckey, editor, Principles and Practice of Constraint Programming, volume 5202 of Lecture Notes in Computer Science, pages 342-357. Springer Berlin Heidelberg, 2008.

[2] Clement Aubry, Rozenn Desmare, and Luc Jaulin. Loop detection of mobile robots using interval analysis. Automatica, 49(2):463 - 470, 2013.

[3] Frédéric Benhamou, Frédéric Goualard, Laurent Granvilliers, and Jean-François Puget. Revising hull and box consistency. In Int. Conf. on Logic Programming. Citeseer, 1999.

[4] G. Chabert and L. Jaulin. Contractor Programming. Artificial Intelligence, 173:1079-1100, 2009.

[5] Vincent Drevelle and Philippe Bonnifait. Localization confidence domains via set inversion on short-term trajectory. 2013.

[6] I. Galton. Analog-input digital phase-locked loops for precise frequency and phase demodulation. Circuits and Systems II: Analog and Digital Signal Processing, IEEE Transactions on, 42(10):621-630, 1995.

[7] Rémy Guyonneau, Sébastien Lagrange, Laurent Hardouin, Philippe Lucidarme, et al. Interval analysis for kidnapping problem using range sensors. In SWIM'11 Small Workshop on Interval Methods, 2011.

[8] L. Jaulin. A Nonlinear Set-membership Approach for the Localization and Map Building of an Underwater Robot using Interval Constraint Propagation. IEEE Transaction on Robotics, 25(1):88-98, 2009.

[9] L. Jaulin. Robust set membership state estimation ; application to underwater robotics. Automatica, 45(1):202-206, 2009.

[10] L. Jaulin. Solving set-valued constraint satisfaction problems. In SCAN 2010, Lyon (France), 2010.

[11] L. Jaulin and E. Walter. Guaranteed nonlinear parameter estimation from bounded-error data via interval analysis. Mathematics and Computers in Simulation, 35(2):123-137, 1993.

[12] M. Kieffer, L. Jaulin, and E. Walter. Guaranteed recursive nonlinear state estimation using interval analysis. In Proceedings of the 37th IEEE Conference on Decision and Control, pages 3966-3971, Tampa, FL, 1998. 
[13] W.C. Lindsey and Chak Ming Chie. A survey of digital phase-locked loops. Proceedings of the IEEE, 69(4):410-431, 1981.

[14] Neil Gordon M. Sanjeev Arulampalam, Simon Maskell and Tim Clapp. A tutorial on particle filters for online nonlinear/non-gaussian bayesian tracking. IEEE Transactions on Signal Processing, 50(2):174$188,2002$.

[15] Keith Ansel Marzullo. Maintaining the time in a distributed system: an example of a loosely-coupled distributed service (synchronization, fault-tolerance, debugging). $\mathrm{PhD}$ thesis, 1984.

[16] D. Meizel, O. Leveque, L. Jaulin, and E. Walter. Initial localization by set inversion. IEEE transactions on robotics and Automation, 18(6):966-971, 2002.

[17] J. Opderbecke. At-sea calibration of a usbl underwater vehicle positioning system. In OCEANS '97. MTS/IEEE Conference Proceedings, volume 1, pages 721-726 vol.1, 1997.

[18] S.M. Smith and D. Kronen. Experimental results of an inexpensive short baseline acoustic positioning system for auv navigation. In OCEANS '97. MTS/IEEE Conference Proceedings, volume 1, pages 714720 vol.1, 1997.

[19] Keith Vickery. Acoustic positioning systems. a practical overview of current systems. In Autonomous Underwater Vehicles, 1998. AUV'98. Proceedings Of The 1998 Workshop on, pages 5-17. IEEE, 1998.

Mohamed Saad IBN SEDDIK

ENSTA Bretagne, 2 Rue Francois Verny, BREST FRANCE.

e-mail: ms.ibnseddik@ensta-bretagne.org

Luc JAULIN

ENSTA Bretagne, 2 Rue Francois Verny, BREST FRANCE.

e-mail: luc.jaulin@ensta-bretagne.fr

Jonathan GRIMSDALE

CGG, 27 Avenue Carnot, MASSY FRANCE.

e-mail: jonathan.grimsdale@cgg.com 